\title{
TADF/RTP OLED organic emitters based on concaved N-PAHs with tunable intrinsic D-A electronic structure
}

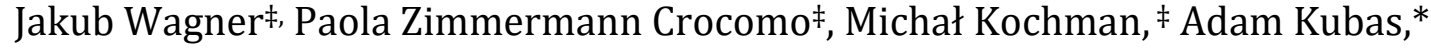 \\ Przemysław Data, ${ }^{*}$ Marcin Lindner*
}

Institute of Organic Chemistry, Polish Academy of Sciences, Kasprzaka 44/52, 01-224 Warsaw, Poland;

Institute of Physical Chemistry, Polish Academy of Sciences, Kasprzaka 44/52, 01-224 Warsaw, Poland;

Silesian University of Technology, Faculty of Chemistry, Department of Physical Chemistry and Technology of Polymers, Strzody

9/208d, 44-100 Gliwice, Poland

‡ these authors contributed equally

KEYWORDS: PAHs, N-doping, Dyes/Pigments, Functional Aromatic Materials, RTP, TADF, OLEDs

\begin{abstract}
Polycyclic aromatic hydrocarbons (PAHs) with centrally positioned nitrogen dopants possess a unique curved structure and strong electron-donating features. However, the lack of tools to synthetically affect their bandgap engineering and charge-transfer (CT) characteristic is detrimental to their future optoelectronics use because of usually low PLQY efficiency. Facing this challenge, we report on developing the first fully conjugated, curved N-PAHs containing phenazine terminus with the D-A electronic structures, which are herein studied as functional optoelectronic material. We evidence the influence of curvature on minimizing HOMO-LUMO overlap, which was severely reflected in small $\Delta \mathrm{E}_{\mathrm{ST}}$ values, indispensable to enhance the RISC rate constant. Within this approach, we evaluate the utility of the concaved system as TADF/RTP emitters which has not been explored so far in the context of non-planar N-PAHs. By variable accepting strength of phenazines employed, the photoluminescence quantum yields ( $\left.\Phi_{\mathrm{PL}}\right)$ were tuned, ranging from the lowest $9 \%$ up to the highest $86 \%$ with dinitrile terminus. As a proof of concept, solid-state OLED devices were constructed, exhibiting yellow to orange emission with the best maximum external EL quantum efficiency (EQE) of 12\% for acceptor built up on 3-(trifluoromethyl)phenyl decorated phenazine that is demonstrated for the first time for curved D-A embedded N-PAHs.
\end{abstract}

Polycyclic aromatic hydrocarbons (PAHs) ${ }^{1-3}$ unveiled within the last two decades have demonstrated an immense potential to be applied in the domain of chemical sensors, ${ }^{4}$ organic field-effect transistors (OFETs), ${ }^{5}$ organic solar cells, ${ }^{6}$ fluorescent labeling, ${ }^{7,8}$ liquid crystals, ${ }^{9,10}$ and organic light-emitting diodes (OLEDs). ${ }^{11}$ One of the most efficient approaches to rationally tune their physicochemical features is the insertion/doping of the main group elements on the periphery, and primarily the center of the polycyclic framework as the replacement of $s p^{2}$-hybridized carbon atoms alter the MO energy levels. In this context, the geometry adopted by PAHs is crucial, which is of paramount importance for nitrogen (N)-doped PAHs. ${ }^{12,13}$ While centrally positioned N-heteroatom is surrounded solely by sixmembered rings, such arrangement leads to the various planar or slightly bent architectures, including carbonyl, dimethyl, $\mathrm{O}$, and $\mathrm{S}$ bridged $\mathrm{N}$-heterotriangulenes, respectively (see Fig. 1a, 1-6). ${ }^{14-17}$ Even though the latter ones prove to be strong electron donors, their modest thermal and morphological stability and vast tendency towards $\pi-\pi$ interaction impede the practical use. Regarding non-planar species, these are being formed when N-dopant is encircled with pentagons and heptagons. Such structurization has led to concaved or bowled-shaped three-dimensional arrangements, ${ }^{12,13}$ which were envisaged to significantly contribute to their photophysical properties.

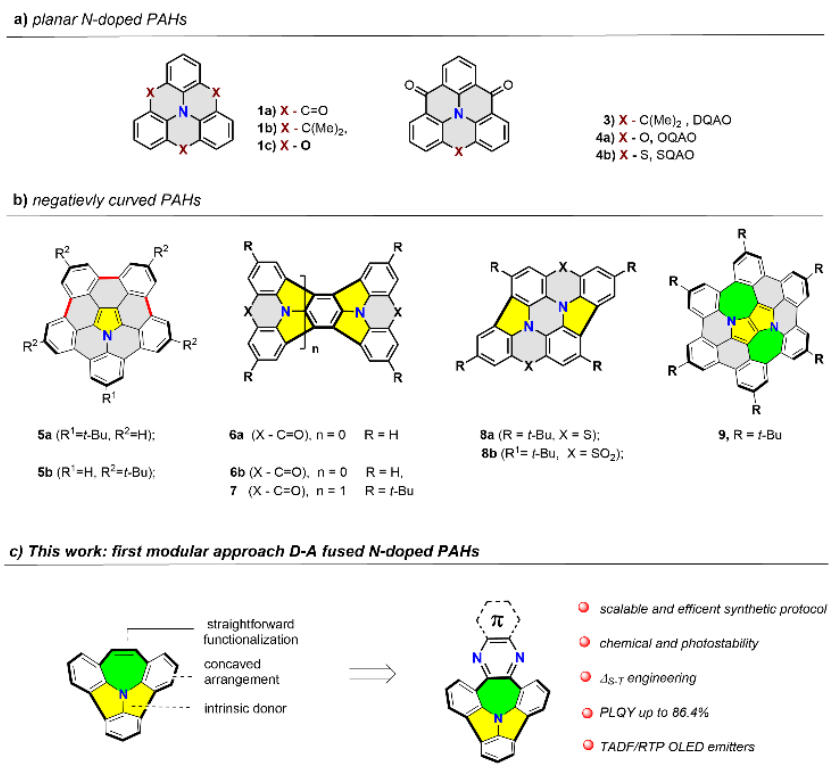

Figure 1. Chemical evolution of the N-doped PAHs

Nevertheless, in sharp contrast to the planar analogs, solely a few examples (see Fig. $1 \mathrm{~b}, \mathbf{7 a}, \mathbf{b}-\mathbf{8}$ ) of $\pi$-extended $\mathrm{N}$-embedded PAHs have been so far denoted as their preparation often requires tedious and multi-step synthetic 
methodologies. Notably, the seminal works on the pyrroleembedded curved PAHs were independently reported by Nozaki and Shinokubo. ${ }^{18,19}$ Consecutively, Wang and coworkers demonstrated acridine(one)s $\mathrm{s}^{20-22}$ and phenothiazine $^{23}$ containing PAHs which structures are displayed at Fig. 1b, 8a,b, 9, 10a,b, as suitable building blocks to ensure desired curvature ending up with concaved and boat-like conformations, respectively. Recently, Gryko's group showed the utility of pyrrolo[3,2- $b]$ pyrrole in forming bucky-bowl system, with inverse Stone-Thrower-Wales defects, first via "on surface" 24 and subsequently "in solution"25 approaches (Fig. 1b, 11). Despite the promising structural alignment of electron-donating group (D), exploration of curved N-PAHs as prospective optoelectronic materials is limited by relatively low emission efficiency. One could anticipate tailoring of their properties, addressed at the modulation of bandgap and S-T levels engineering, via post-modification of the periphery of the $\pi$ scaffold. Nonetheless, there is a need for mounting solubilizers in usually the most reactive position of N-PAH rings, which prevents further synthetic transformations with electron-accepting species (A). Therefore, the rational design of nonplanar thoroughly conjugated N-PAHs with a D-A electronic structure that compensates for the desired functions and synthetic availability has remained elusive.

Thermally activated delay fluorescence (TADF) is a unique photophysical phenomenon and is promising for enhancing external quantum efficiency (EQE) of organic lightemitting diodes (OLEDs). TADF-active organic emitters can theoretically harvest $100 \%$ excitons generated in the emitting layer by electrical excitation and convert them into light through efficient reverse intersystem crossing (rISC). ${ }^{26-29}$ The key issue in designing efficient TADF emit- ters is the acceleration of a spin-forbidden and endothermic rISC process $\left(\mathrm{T}_{1} \rightarrow \mathrm{S}_{1}\right)$. D-A $\pi$-conjugated systems with a sizeable D-A dihedral angle and a vibrational motion can provide a solution by minimizing the singlet-triplet energy gap $\left(\Delta \mathrm{E}_{\mathrm{ST}}<0.2 \mathrm{eV}\right)$ to lower the activation energy for rISC and mixing excited CT and LE states to allow spin-flip electronic transitions. Therefore, the development of TADF active organic materials has been vastly relied on planar and branched D-A species. These molecules possess certain drawbacks linked with significant oscillator strengths, specifically small radiative emission cross-sections. This effect adversely influences photoluminescence quantum yield (PLQY.). On the other hand, when moderate $\Delta \mathrm{E}_{\mathrm{ST}}$ $(0.3-0.6 \mathrm{eV})$ is identified, the competitive to TADF, photons emission through room-temperature phosphorescence $(\mathrm{RTP})^{30}$ can occur. Consequently, the molecular design targeted strictly at TADF or RTP is fundamentally important from the viewpoint of future applications in domains of sensors, data encryption, and white-emitting OLEDs (WOLEDs). ${ }^{31}$

Herein, we report a concise and efficient synthetic strategy towards a new type of N-PAHs with intrinsic D-A electronic structures, which were for the first time handled as yellow to orange TADF/RTP OLED emitters (Fig. 1c). The proposed motif bears spatially separated HOMO-LUMO gaps through an antiaromatic seven-membered ring (see SI) that plays a prominent role in delivering attractive photophysical properties. An entirely fused system compromises moderate to weak oscillator strength $(f)$ for HOMO $\rightarrow$ LUMO transitions accompanied with small $\Delta_{\text {S-T }}$ energy. In contrast, the strength of the acceptor contributes to the remarkable PLQY amplification up to $86 \%$ de-
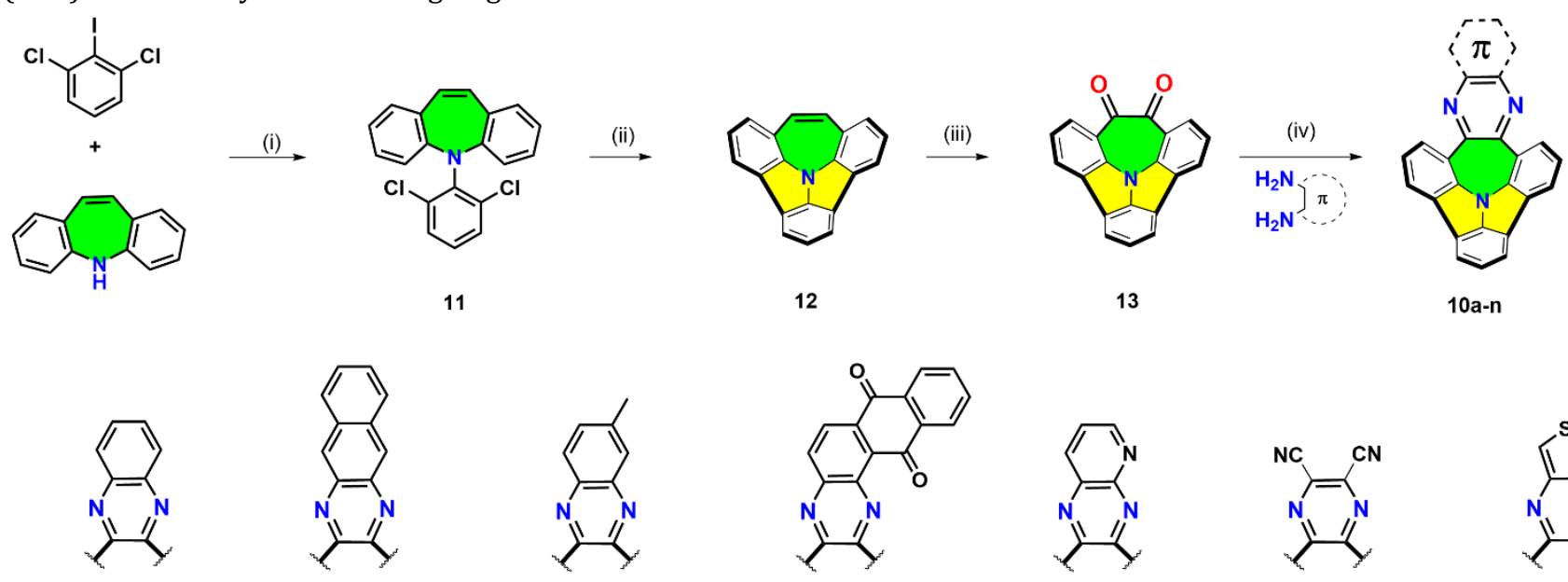

$10 \mathrm{c}$
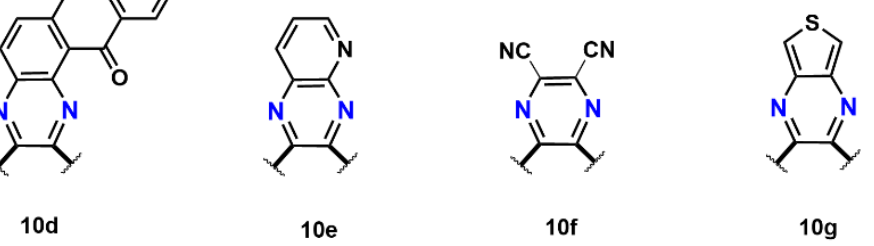

$10 \mathrm{a}$

$10 \mathrm{~b}$
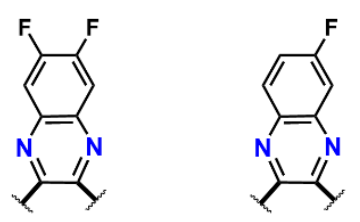

$10 \mathrm{e}$

$10 f$

$10 \mathrm{~g}$

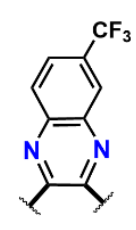

$10 \mathrm{~h}$

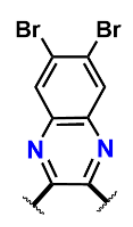

$10 \mathrm{i}$

$10 \mathrm{j}$

$10 \mathrm{k}$

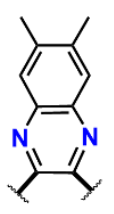

101

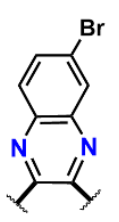

$10 \mathrm{~m}$ 10a-n

Reagents and conditions: (i) $\mathrm{Pd}(\mathrm{OAc})_{2}, \mathrm{P}(\mathrm{tBu})_{3} \mathrm{HBF}_{4}, \mathrm{NaOtBu}$, toluene; $89 \%$ (ii) $\left[\mathrm{Pd}(\mathrm{Pcy})_{3}\right] \mathrm{Cl}_{2}, \mathrm{DBU}, \mathrm{DMAc}, \mathrm{MW}, 77 \%$; (iii) $\mathrm{RuCl}, \mathrm{NalO}_{4}, \mathrm{NMI}$ THF/DCM/ $\mathrm{H}_{2} \mathrm{O}, 37 \%$ or BSA o-dichlorobenzene, $65 \%$ (v) $\mathrm{AcOH} / \mathrm{EtOH}, 1: 1,56-79 \%$

Scheme 1 The synthetic path towards D-A arranged N-PAHs with an array of electronically diverse phenazines 
termined for 10f. Thus, the OLEDs fabricated with the developed N-PAHs 10a-10n as TADF/RTP emitters can achieve an EQE as high as $12 \%$ along with satisfactory operational stability and a low roll-off process

\section{Results and discussion}

\section{Molecular design and synthesis}

To induce the anticipated curvature in the PAH system, our molecular design strategy involved placing two pentagons and one heptagon around the central nitrogen atom (Scheme 1). On the other hand, using a seven-membered ring with unsaturated termini of azepine ${ }^{32}$ would allow one to oxidize it to diketone 13. This type of intermediate is known to undergo a smooth condensation with various 1,2-aromatic diamines providing $\pi$-extended scaffold of phenazines. Accordingly, the latter moieties are a wellspread class of efficient electron acceptors employed so far in U-shaped, macrocyclic, and flat TADF OLED emitters. ${ }^{33}$. Therefore, utilization of such moieties with electronically varied substituents would allow us to gain strict control of the strength of acceptor. With such molecular engineering, we anticipate shaping optoelectronic features of our concaved N-PAHs with a great emphasis on excited states and bandgap energies.

The synthetic pathway towards a set of concaved dyes 10a10m starts from the chemoselective Hartwig - Buchwald amination $^{34}$ of commercially available dibenz $[b, f]$ azepine and 2,6-dichloro-1-iodobenzene delivering an amine 11 in excellent $89 \%$ yield as depicted in Scheme 1. The subsequent step involves the annulation of two 5 -membered rings by microwave-assisted direct arylation. By modifying Siegel's conditions $^{35}$ (only $5 \mathrm{~mol} \%$ of Pd-catalyst was implemented), dichlorinated precursor was subjected to heating for $40 \mathrm{~min}$ to obtain compound $\mathbf{1 2}$ with a very good $77 \%$ yield on a multigram scale. With this intermediate in hand, we further pursued the formation of diketone 13. We first examined the approach commonly used for selective oxidation of pyreneembedded PAHs, ${ }^{36}$ and the synthesis of the key intermediate 13 was succeeded in satisfying $37 \%$ yield. With the use of benzeneseleninic acid anhydride (BSA), 37 we could significantly improve the oxidation efficiency up to $65 \%$ yield. In the final step, a series of acid-promoted condensations were conducted to provide a set of phenazines that differed in the acceptors' strength. To our delight, we were able to assemble 14 electronically diverse species 10a-n in yields ranging from moderate to very good (pointed in Scheme 1Fig 1.). We indeed found each step to be readily scalable, whereas three of them (see SI) could be handled without a CC separation that could raise the prospective applicability of the current approach.

\section{Single-Crystal X-ray Crystallographic Analyses.}

Single crystals of $\mathbf{1 0 1}$ suitable for X-ray analysis were obtained by their evaporation from the mixture of solvents dichloromethane/tetrahydrofuran at room temperature. This compound crystallizes in the P-1 space group with two molecules per unit cell. The X-ray crystallographic analysis, displayed in Fig. 2, unambiguously confirmed the desired concaved geometry. The distance of the central nitrogen atom and the plane of the three carbon atoms of the peripheral benzene rings indicate the depth to be $0.63 \AA$ (Fig. 2b). Although the three $\mathrm{N}-\mathrm{C}$ bond lengths oscillate around 1.36-1.38 , as for $\mathbf{8 a}$ (Fig. 1b), the lower depth of $\mathbf{1 0 1}$ compared to $\mathbf{8 a}$ could be reasoned by a minor strain produced by seven-membered rings. This finding

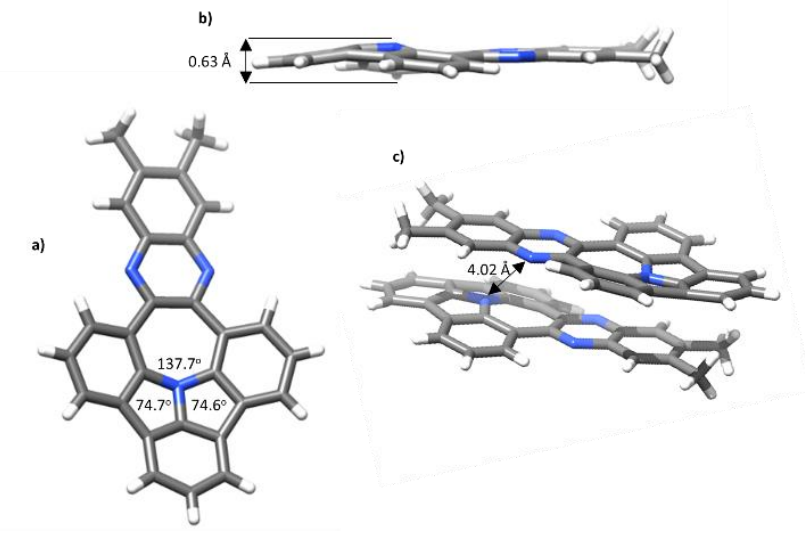

Figure 2. Crystal structure of 10l; a) top view of the crystal structure of 10l; b) the molecular packing of $\mathbf{1 0 l}$ in the crystal; c) side view of the crystal $\mathbf{1 0 1}$

can be easily tracked with the value measured for $\mathrm{C}-\mathrm{N}-\mathrm{C}$ bond angles $137.7^{\circ}, 74.7^{\circ}$, and $74.6^{\circ}$ (Fig. 2a), whereas 8a possessing six-membered ring with a larger constrain exhibits $121.6^{\circ}, 107.8^{\circ}$, and $108.7^{\circ}$. Contrary to the previously reported curved 7-9, which nearly all stack in a concave-convex manner assembled in the same direction, the obtained solidstate structure favors a "head-to-tail" antiperiplanar arrangement. As is shown in Fig 2c, tertiary amines are localized as opposed to each other with the almost parallel orientation of D-A units. This observation is manifested by short distances of $4.02 \AA$ between nitrogen's electron-rich and -deficient atoms. The predominant arrangement of neighboring molecules could favor the formation of excitons, which is beneficial from the viewpoint of photophysics and the further possible TADF/RTP OLED applications.

\section{Quantum chemical calculations}

The optical properties of 10a-10n were first examined theoretically with the second-order algebraic diagrammatic construction theory with spin-component scaling (SCSADC(2)). ${ }^{38,39}$ For the sake of clarity, here we focus on $\mathbf{1 0 l}$ as a representative example of this class of compounds. Outcomes for the other compounds are presented in the Supporting Information.

The calculated vertical excitation energies and associated oscillator strengths for $\mathbf{1 0 l}$ are given in Table 1. Accompanying this data, in Figure 2 we show plots of natural transition orbitals (NTOs) ${ }^{40}$ for transitions from the ground state into the two lowest singlets and the two lowest triplet excited states. According to our calculations, the lowest singlet excited state $\left(S_{1}\right)$ of $\mathbf{1 0 1}$ is a $\pi \pi^{*}$-type state with vertical excitation energy of $3.455 \mathrm{eV}$. The inspection of the predominant hole-particle NTO pair for the $S_{0} \rightarrow S_{1}$ transition (Figure 2a) shows that the $S_{1}$ state has a chargetransfer character that arises from the excitation of an electron from a $\pi$ orbital that is distributed mainly on rings VI, VII, and VIII into a $\pi^{*}$ orbital that is localized on rings VII and VIII. This transition is characterized by an appreciably large oscillator strength, considerably more significant than the transitions into the other low-lying singlet excited state. Thus, the lowest photo-absorption band of 
101 can be assigned mainly to the transition into the $S_{1}$ state.

Table 1. Vertical excitation energies $(\Delta E)$ and associated oscillator strengths $(f)$ of the representative compound 101 calculated at the SCS-ADC(2) level of theory. The calculation was performed at the ground-state equilibrium geometry optimized at the SCS-MP2 level.

\begin{tabular}{lll} 
State & $\Delta E[\mathrm{eV}]$ & $f$ \\
\hline $\mathrm{S}_{1}\left({ }^{1} \pi \pi^{*}\right)$ & 3.455 & 0.3540 \\
$\mathrm{~S}_{2}\left({ }^{1} \pi \pi^{*}\right)$ & 3.522 & 0.0361 \\
$\mathrm{~S}_{3}\left({ }^{1} \pi \pi^{*}\right)$ & 3.870 & 0.0100 \\
$\mathrm{~S}_{4}\left({ }^{1} \mathrm{n} \pi^{*}\right)$ & 4.061 & 0.0033 \\
$\mathrm{~T}_{1}\left({ }^{3} \pi \pi^{*}\right)$ & 2.984 & 0 \\
$\mathrm{~T}_{2}\left({ }^{3} \pi \pi^{*}\right)$ & 3.091 & 0 \\
$\mathrm{~T}_{3}\left({ }^{3} \pi \pi^{*}\right)$ & 3.398 & 0 \\
$\mathrm{~T}_{4}\left({ }^{3} \pi \pi^{*}\right)$ & 3.419 & 0
\end{tabular}

The $S_{1}$ state is closely followed by the $S_{2}$ state with vertical excitation energy of $4.522 \mathrm{eV}$. The $\mathrm{S}_{2}$ state involves the excitation of an electron from a $\pi$ orbital localized on rings II, III, IV, and V into a $\pi^{*}$ orbital localized on rings I, II, III, $\mathrm{IV}$, and $\mathrm{V}$. The transition into the $\mathrm{S}_{2}$ state displays a relatively low oscillator strength. Unlike the $S_{1}$ state, the $S_{2}$ state does not exhibit a significant charge-transfer character. $S_{3}$ and $S_{4}$ states are energetically well-separated from the $S_{1} / S_{2}$ states and display small oscillator strengths for transitions out of the ground state.

In the other compounds in the series 10a-10n, the electronic structures of the $S_{1}$ and $S_{2}$ states are qualitatively similar as in 10l. The exception is $\mathbf{1 0 f}$, for which the ordering of the lowest two singlet states is inverted: in 10f, the dark ${ }^{1} \pi \pi^{*}$ state lies vertically below the bright ${ }^{1} \pi \pi^{*}$ state.

The lowest two triplet states $\left(\mathrm{T}_{1}\right.$ and $\left.\mathrm{T}_{2}\right)$ are found at 2.984 $\mathrm{eV}$ and $3.091 \mathrm{eV}$, respectively. According to the NTO analysis, both states exhibit pronounced multiconfigurational character: the $S_{0} \rightarrow T_{1}$ and the $S_{0} \rightarrow T_{2}$ transitions have significant contributions from two hole-particle NTO pairs. This is presumably because the $T_{1}$ and $T_{2}$ are close in energy and interact strongly. We also located $\mathrm{T}_{3}$ and $\mathrm{T}_{4}$ states higher in energy, ca. $0.4 \mathrm{eV}$ above the $\mathrm{T}_{2}$ state.

In all cases, the first triplet state was found lower in energy than first excited singlet state. However, the energy difference is rather small and yield on average $0.46 \mathrm{eV}$ for the entire set. The lowest difference was identified for compound $10 \mathrm{~d}(0.24 \mathrm{eV})$. Although the calculations were carried out in vacuum and for vertical excitations only, obtained relatively low $\mathrm{T}_{1}-\mathrm{S}_{1}$ energy differences suggest that crystal packing, interaction with solvent molecules or vibrational motion may influence the photophysics of examined systems significantly.
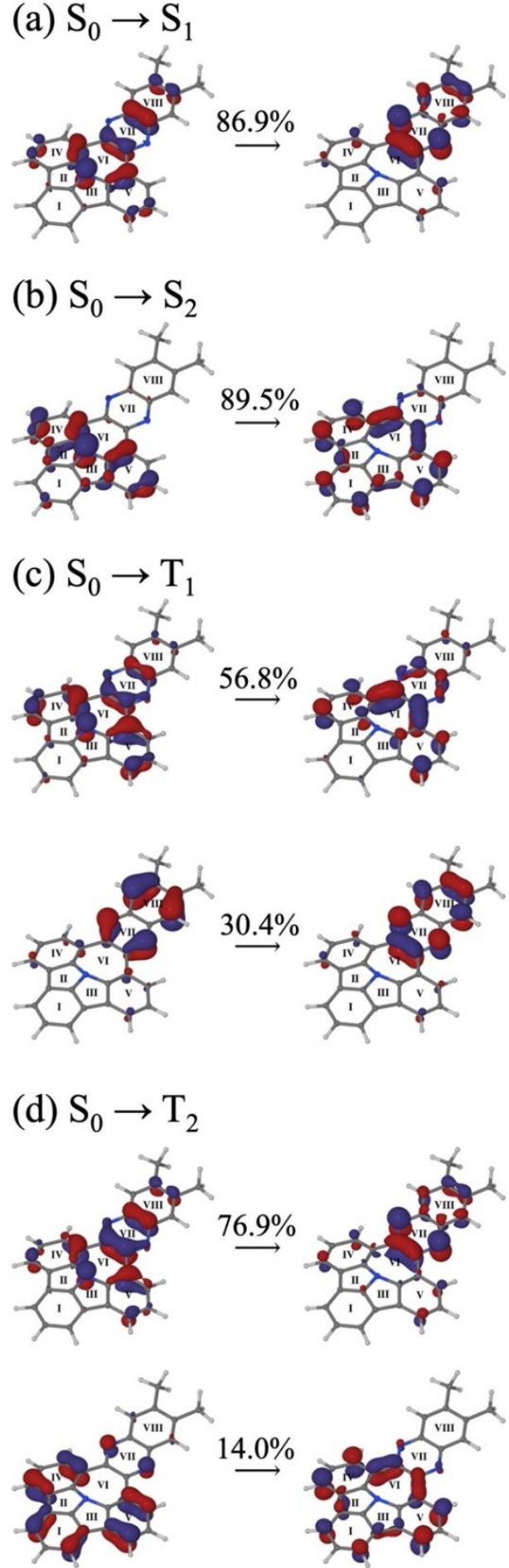

Figure 2. Dominant NTOs for vertical transitions of $\mathbf{1 0 1}$ (SCSADC(2)) plotted as isosurfaces with isovalues of $\pm 0.05 \mathrm{a}_{0}^{-3 / 2}$. The eigenvalue $\left(\lambda_{i}\right)$ for each hole-particle NTO pair is given in terms of a percentage contribution. The rings are numbered with Roman numerals.

\section{Photophysics}

In the first step of optical measurements, the basic absorption and fluorescence spectra were collected to estimate the influence of structure on excitation and emission properties with respect to electronic character of acceptor. In 
all cases, we observed emission from the ${ }^{1} \mathrm{LE}$ in toluene and ${ }^{1} \mathrm{CT}$ in more polar dichloromethane (DCM) and tetrahydrofuran (THF). Interestingly, there is no dramatic bathochromic shift related to the increase of polarity of the solvent. That could suggest the mixed ${ }^{1} \mathrm{LE} \&{ }^{1} \mathrm{CT}$ character. The only significant shift was observed for the 10d compound with anthraquinone unit in DCM. As for the structural influence, there is a significant bathochromic shift of emission between 10a and 10b, related to the increase of conjugation in the structure due to the additional benzene ring. There is no significant influence by a methyl group (10c, 10l), but the insertion of a nitrogen atom (10e) shifts the emission to lower energies (Figure 6). The highest bathochromic shift was observed due to the addition of two nitrile groups (10f). The impact of the halogen group is less impressive with the highest shift for double bromine atom side groups (10i), which is two times stronger than mono bromine substitution (10m), similar behavior was observed for fluorine side groups (10j, 10k), Figure 6).
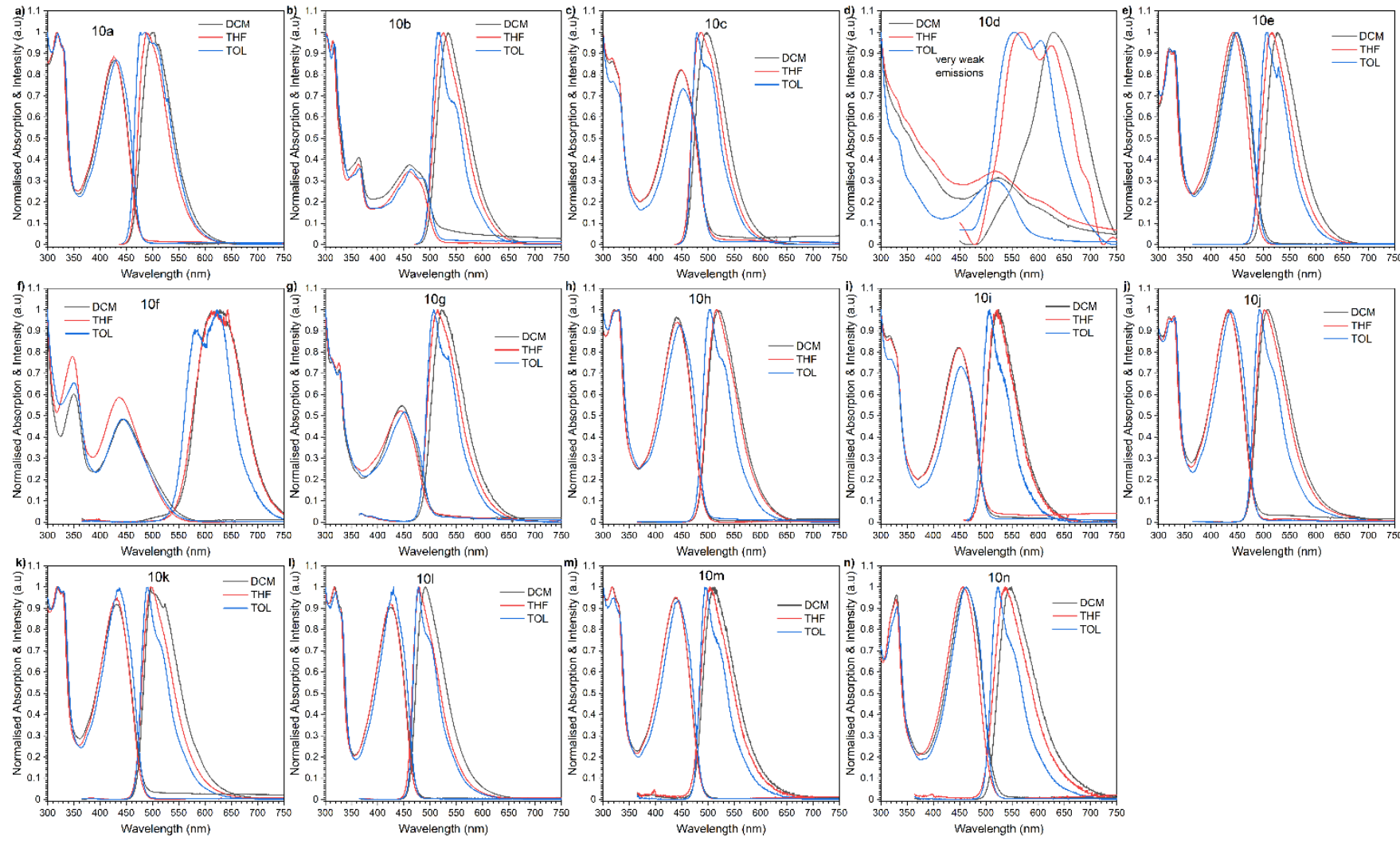

Figure 6. UV-vis absorption and PL spectra of 10a-n (c 10-5 M) diluted solutions in dichloromethane, tetrahydrofuran, and toluene solvents.

As the next step, the synthesized compounds were investigated in the solid-state to observe the possible behavior as the active materials in organic light-emitting diode (OLED) structure. Two matrices were chosen to avoid the possible aggregation, polymer Zeonex as the stage between solution and solid-state and 4,4'-bis( $N$-carbazolyl)-1,1'-biphenyl (CBP) as the host for OLED. It could be noticed that the fluorescence emission (maximum peak) is similar in the Zeonex matrix as it was in the solutions (Figure 7). On the other hand, a solid matrix allows us to observe strong delay emission. Depending on the substitution, the thermally activated delayed fluorescence (TADF), roomtemperature phosphorescence (RTP), or dual TADF \& RTP was observed. Theoretically, to obtain efficient TADF emission, our compound should have as close as possible the singlet and triplet excited states. There are several ways to estimate singlet and triplet excited states, the onset of the respective emission or the maxima of the emissions are used. In our case, to allow for the comparison with theoret- ical calculations, we decide to use emission maxima. In our case, the lowest $\Delta \mathrm{E}_{\text {sT }}$ gaps were observed for compounds with additional benzene ring (10b), thiophene ring $(\mathbf{1 0 g})$ and double bromine group (10i). In the two compounds (10b, 10i), the low $\Delta \mathrm{E}_{\text {sт }}$ gap was connected with the same effect like in solution (an increase of conjugation, decrease the charge transfer energy). The groups affect the ${ }^{1} \mathrm{CT}$ energy state, where the triplet localized energy state remains ( $\left.{ }^{3} \mathrm{LE}\right)$. In the case of the derivative with thiophene unit $(\mathbf{1 0 g})$, it seems that the group is affecting the triplet energy state, which is one of the highest (Figure $7 \mathrm{~g}$ ). To prove, which actual emission process we observe, the emission of the compounds at different delayed times were collected and compared (Figure S2). Moreover, the emission spectra were obtained at different temperatures during the time-resolved photoluminescence measurement to estimate where is the phosphorescence and the point when the thermal activation occur. To decide if the compound exhibit emission through the TADF or RTP process, 
we need to compare ns range emission with at least $\mu$ s one at $300 \mathrm{~K}$ and the phosphorescence emission. In our case, most of the compounds in the Zeonex matrix exhibit TADF emission with certain deviations. Compounds 10d,e,g,h,n had pure TADF emission, where delayed emission $(80 \mathrm{~ms}$, $300 \mathrm{~K}$ ) matches the prompt ns emission. In the case of $\mathbf{1 0 a}, \mathbf{b}, \mathbf{c}, \mathbf{f}, \mathbf{i}, \mathbf{l}$, we have mixed TADF\&RTP emission where RTP contribution is higher than $1 \%$ and for compounds $\mathbf{1 0 j}, \mathbf{k}$, the RTP contribution is lower than 1\%. Only compound $10 \mathrm{~m}$ with a single bromine group had pure RTP emission at $80 \mathrm{~ms}$ delay time (Figure $7 \mathrm{~m}$ ). Very little change like insertion of nitrogen group (10n), change the emission to pure TADF or addition of second bromine group (10i)creates mixed TADF\&RTP emitter.

Nonetheless, the most intriguing behavior was observed for 10d and 10f. Insightful analysis of obtained dates could hint an appearing of the inversion of singlet and triplet energy states in both cases. In the case of $\mathbf{1 0 d}$, it may not be so obvious, but the prompt fluorescence emission is a set of two signals, one with maxima at $494 \mathrm{~nm}$ and the second at $590 \mathrm{~nm}$. The signal at 494 disappear around 10 ns delay time, and a single $590 \mathrm{~nm}$ remain. That means our lowest singlet state is $590 \mathrm{~nm}(2.10 \mathrm{eV})$ and our real
$\Delta \mathrm{E}_{\mathrm{ST}}=-0.25 \mathrm{eV}$ (Figure $7 \mathrm{~d}$ ). Similar but more visible observation is identified for $\mathbf{1 0 f}$, the triplet energy state is higher by $0.34 \mathrm{eV}$ from the lowest singlet excited state (Figure $7 f)$. It has to be noticed, however, that experimental values are not in accordance with those extracted from ab-initio performed computations. The origin of negative $\Delta \mathrm{E}_{\mathrm{ST}}$ remains an open question and an additional, more targeted molecular design is indispensable to clearly elucidate observed phenomena. Nevertheless, that is not all, in the case of $\mathbf{1 0 d}$, as delayed emission, we observe TADF but not from $S_{1}$ energy state but higher one $\left(S_{2}\right)$. Such experimental finding is consistent with calculations (for more details see SI) made for compound $\mathbf{1 0 d}$ as significant $f$ value ("bright" oscillator) for $S_{2}$ state was determined. In the case of the 10f compound, we observe mixed emissions based on TADF and RTP processes. In most of the cases, the emission developed at $\mu$ s regime and (ISC/rISC) cycle till ms times. Nevertheless, if we would look closely to the RTP emitter $(\mathbf{1 0 m})$, we can notice that at $\mu$ s the emission if due to TADF process not RTP, the emission start shifting at around $0.2 \mathrm{~ms}$ and pure RTP is observed (Figure S2m). Similar observation could be found in $\mathbf{1 0 f}$ compound, in the $\mu$ s delay time we observe only TADF and mixed TADF\&RTP in ms delay time (Figure S2f).
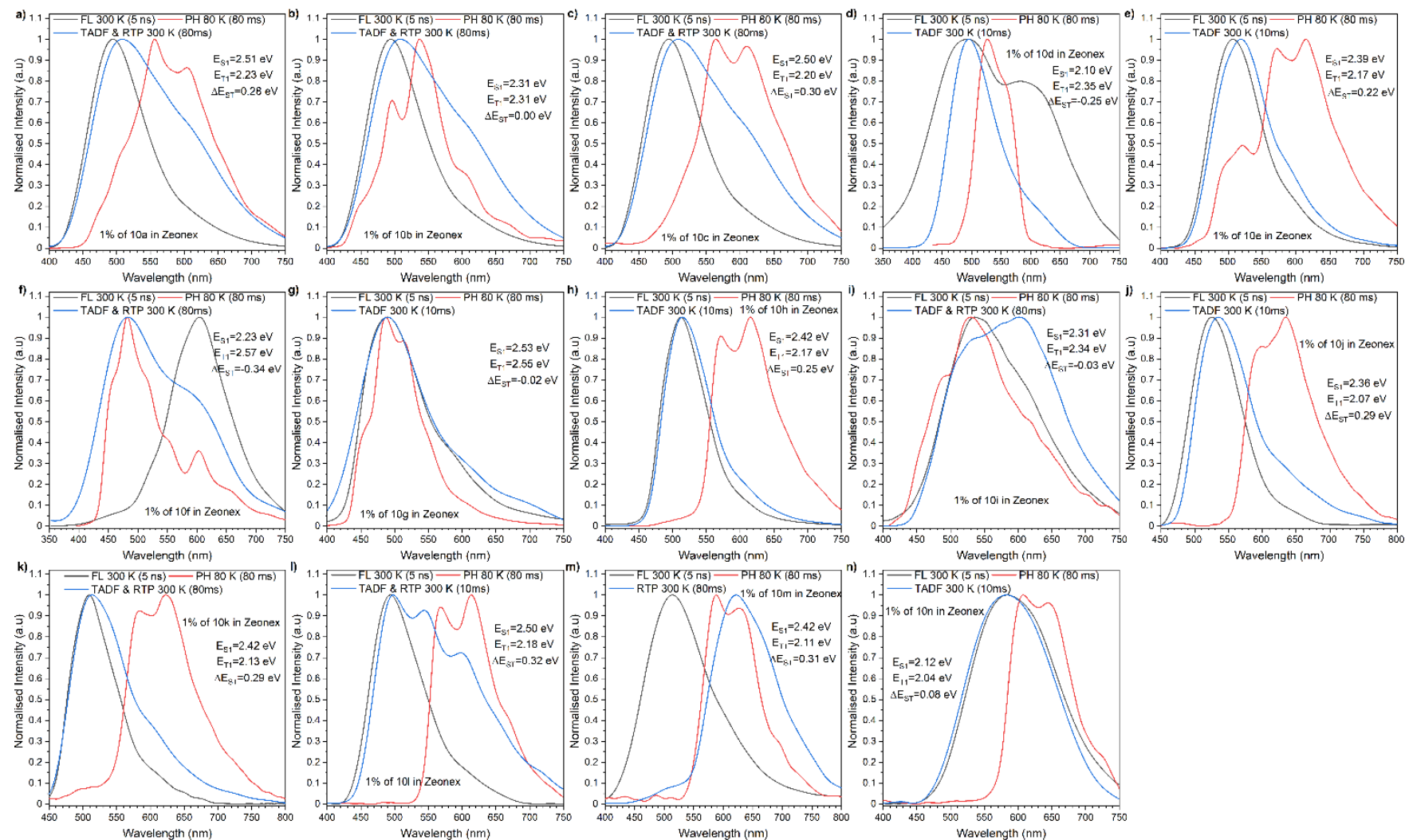

Figure 7. Time-Resolved Spectra of compounds 10a-n in Zeonex matrix obtained during the intensity vs delay time measurement (Figure S2). The energies correspond to the maximum emission peaks.

To evaluate the emissive properties of the compounds as the materials for OLEDs, the photophysics of the emitters in the CBP matrix were conducted (Figure 8, Figure S3). Analyzing the emission in CBP matric, different properties can be noticed in comparison to Zeonex. Comparing emission at ms delay time, compounds $\mathbf{1 0 a}, \mathbf{e}, \mathbf{f}, \mathbf{h}, \mathbf{i}, \mathbf{j}, \mathbf{k}, \mathbf{l}, \mathbf{l}$ n show pure TADF emission, 10c,m shows mixed emission and $\mathbf{1 0 b}, \mathbf{d}, \mathbf{g}$ have RTP. The matrix significantly affects the 10d with anthraquinone and shifts from mixed emission to RTP one, but what is really interesting, in both cases (Zeonex, CBP), the emission from the lowest state is not visible and delay times. That means rISC process is blocked from pass- 
ing to lowest state but the excited state follows to higher singlet state (Zeonex) or stay on lowest triplet energy state (CBP). As for the 10g, the pure TADF in Zeonex changes to pure RTP in CBP. If we look at respective energy levels (Table 2), for $\mathbf{1 0 g}$, both singlet and triplet energies are lowered, but the triplet state is more affected, increasing the $\Delta \mathrm{E}_{\text {sT }}$ up to $0.20 \mathrm{eV}$. This is enough for the TADF process to be turned off and RTP promoted. The process in $10 \mathrm{~b}$ is a little bit different, if we look at the emission spectra development over delay time (Figure S3b), TADF emission starts to be visible at $\mu$ s range and circles over the ISC/rISC process to ms delay times where the RTP process is involved, For compound $\mathbf{1 0 b}$, the shift from TADF to pure RTP is around $0.2 \mathrm{~ms}$ delay time (Figure S3b). Because of the long-lived triplet state, probably in $\mathbf{1 0 b}$ based OLEDs, we would observe only TADF emission. For the other RTP emitters, 10d and $\mathbf{1 0 g}$, the RTP is observed already at $\mu$ s delay times, suggesting that it should work as RTP emitters in OLEDs. Similarly, to the Zeonex matrix, we could observe singlet-triplet inversion in the same two compounds (10d and 10f), but the resulting effect is the opposite. In the case of $\mathbf{1 0 d}$ in Zeonex, we observe TADF character, where in CBP, the emission shift to RTP. In the 10f, where in Zeonex we had dual TADF/RTP emission, in CBP, we observe pure TADF even at longest delay times. For all compounds which had $\Delta \mathrm{E}_{S \mathrm{ST}}$ in Zeonex close to 0 , the gap significantly rose by at least $0.15 \mathrm{eV}$. For the others, the change of the gap was much smaller in the range of $0.05 \mathrm{eV}$ to lower values.
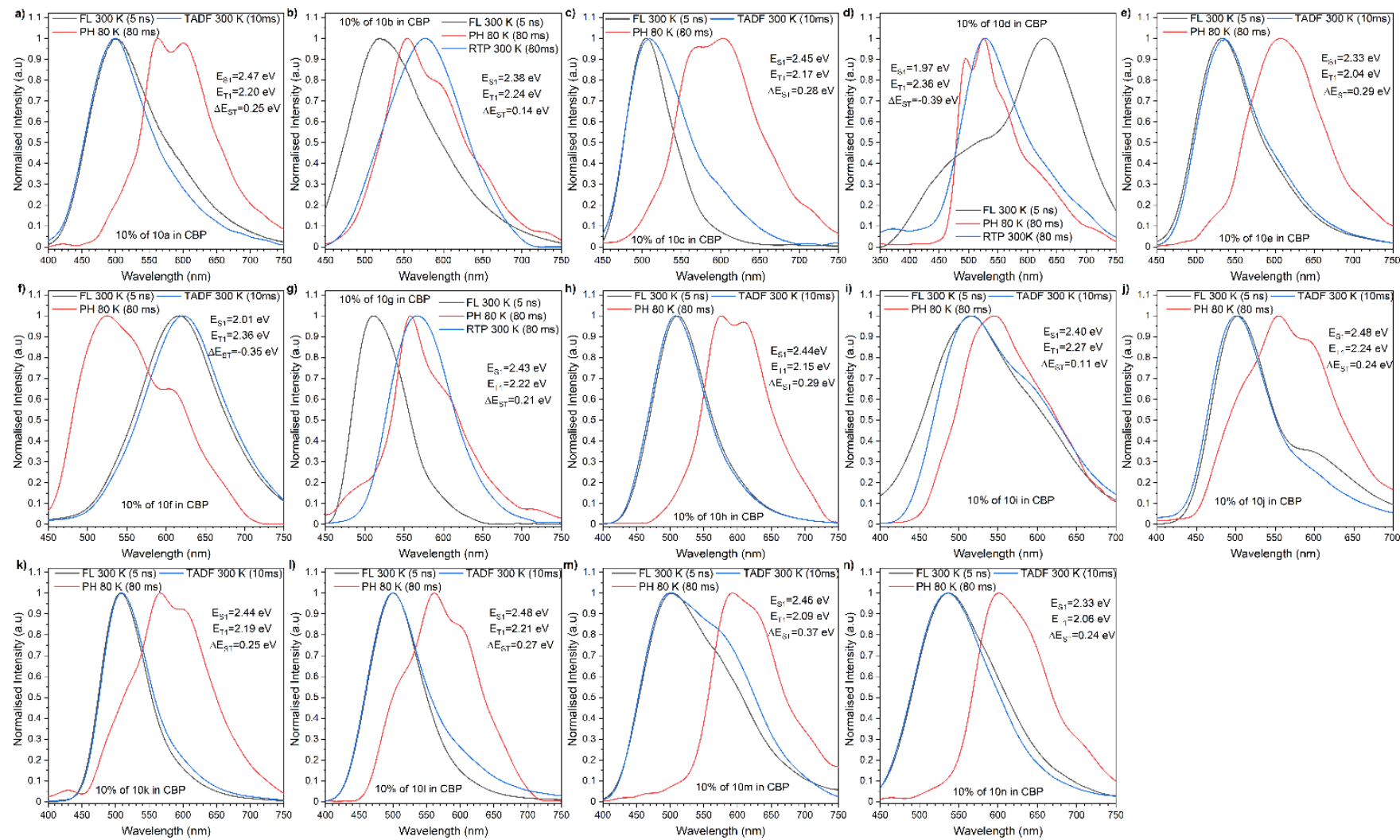

Figure 8. Time-Resolved Spectra of compounds 10a-n in CBP matrix obtained during the intensity vs delay time measurement (Figure S3). The energies correspond to the maximum emission peaks.

One of the most important factors describing the TADF impact on the final device is the DF/PF ratio, this factor tells us how much triplet state contribute to the final emission. As it was stated by Dias et al. ${ }^{41}$ above 3 already give us $100 \%$ use of generated triplets. In the Zeonex matrix, the $10 \mathrm{~b}$ compound showed the highest DF/PF (21.49), nevertheless, above the threshold are also $\mathbf{1 0 e}, \mathbf{h}, \mathbf{i}, \mathbf{j}, \mathbf{k}, \mathbf{l}, \mathbf{n}$, which show the high impact of nitrogen insertion and halogen group to the rISC process. Unfortunately, in all cases but four (10f,h,m,n), we observe a decrease in the $\mathrm{DF} / \mathrm{PF}$ ratio in the CBP matrix. In three $(\mathbf{1 0 h}, \mathbf{m}, \mathbf{n})$, the ratio is still above the threshold, and two (10f,l) at high value ca. 2. As for the RTP emitters $(\mathbf{1 0 d}, \mathbf{g})$, in both cases, the overall RTP emission is much smaller than the fluorescence $(0.43$ and 0.18$)$.

\section{Fabrication and characterization of OLEDs devices}

As the final stage, the OLED devices were fabricated and characterized (Figure 9). The behavior of the emitters in the CBP host was evaluated. The HOMO-LUMO values obtained from electrochemical measurement was used to evaluate the possible OLED device structures. The optimal device structure for the emitters was used in configuration: Devices 10a-n -ITO/NPB [ $N, N^{\prime}-\operatorname{di}\left(1-\right.$ naphthyl)- $N, N^{\prime}$ diphenyl-(1,1'-biphenyl)-4,4'-diamine] (40 nm)/TSBPA [4,4'-(diphenylsilanediyl)bis( $N, N$-diphenylaniline $)]$ $\mathrm{nm}) / 10 \%$ of $\mathbf{1 0 a}-\mathbf{n}$ in CBP $(20 \mathrm{~nm}) / \mathrm{TPBi}\left[2,2^{\prime}, 2^{\prime \prime}-(1,3,5-\right.$ 
benzinetriyl)-tris(1-phenyl-1- $H$-benzimidazole)]

$(50$ $\mathrm{nm}) / \mathrm{LiF}(1 \mathrm{~nm}) / \mathrm{Al}(100 \mathrm{~nm})$ (Figure 9). All the devices fabricated with the emitters but 10d,g showed electrolu- minescence which could be associated with TADF, whereas 10d and $10 \mathrm{~g}$ had RTP emission, which is supported by photophysical analysis.

Table 2. Summary of the general photophysical properties of the compounds.

\begin{tabular}{|c|c|c|c|c|c|c|c|c|c|c|}
\hline Compound & $\begin{array}{l}\lambda_{\mathrm{em}} \\
\mathrm{nm}^{\mathrm{a}}\end{array}$ & Host & $\begin{array}{c}\text { PLQY } \\
(\%)^{b}\end{array}$ & $\begin{array}{l}\tau_{\mathrm{PF}}, \\
\mathrm{ns}^{\mathrm{c}}\end{array}$ & $\begin{array}{l}\tau_{D F}, \\
\mu s^{d}\end{array}$ & $\begin{array}{l}\tau_{\mathrm{Ph}}, \\
\mathrm{ms}^{\mathrm{d}}\end{array}$ & $\mathrm{DF} / \mathrm{PF}^{\mathrm{e}}$ & $\begin{array}{c}S_{1} \\
e^{f}\end{array}$ & $\begin{array}{l}T_{1}, \\
e V^{f}\end{array}$ & $\begin{array}{c}\Delta E_{S T} \\
\text { eVg }\end{array}$ \\
\hline \multirow{2}{*}{$10 a$} & 493 & Zeonex & 10.3 & $3.89 \pm 0.15$ & $10.81 \pm 0.94$ & $1.80 \pm 0.10$ & 1.01 & 2.51 & 2.23 & 0.28 \\
\hline & 502 & CBP & 17.4 & $5.14 \pm 0.19$ & $3.55 \pm 0.34$ & - & 0.20 & 2.47 & 2.20 & 0.26 \\
\hline \multirow{2}{*}{$10 b$} & 536 & Zeonex & 35.8 & $5.22 \pm 0.37$ & $1.82 \pm 0.12$ & $9.23 \pm 0.83$ & 21.49 & 2.31 & 2.31 & 0.00 \\
\hline & 519 & $\mathrm{CBP}$ & 44.8 & $7.82 \pm 0.25$ & $3.52 \pm 0.38$ & $1.21 \pm 0.18$ & 0.14 & 2.38 & 2.24 & 0.15 \\
\hline \multirow{2}{*}{$10 c$} & 497 & Zeonex & 8.4 & $4.96 \pm 0.23$ & $2.47 \pm 0.12$ & $5.97 \pm 0.57$ & 2.80 & 2.50 & 2.20 & 0.30 \\
\hline & 505 & $\mathrm{CBP}$ & 16.1 & $3.87 \pm 0.18$ & $1.63 \pm 0.16$ & - & 0.20 & 2.45 & 2.17 & 0.28 \\
\hline \multirow{2}{*}{$10 d$} & 497 & Zeonex & 1.7 & $3.04 \pm 0.04$ & $7.43 \pm 0.65$ & - & 0.46 & 2.10 & 2.35 & -0.25 \\
\hline & 456 & $\mathrm{CBP}$ & 16.2 & $15.68 \pm 0.91$ & $2.42 \pm 0.30$ & - & 0.43 & 1.97 & 2.36 & -0.39 \\
\hline \multirow{2}{*}{$10 \mathrm{e}$} & 519 & Zeonex & 36.4 & $6.95 \pm 0.38$ & $4.30 \pm 0.49$ & - & 8.17 & 2.39 & 2.17 & 0.22 \\
\hline & 519 & $\mathrm{CBP}$ & 37.0 & $5.32 \pm 0.17$ & $1.58 \pm 0.20$ & - & 0.37 & 2.33 & 2.04 & 0.29 \\
\hline \multirow{2}{*}{$10 f$} & 555 & Zeonex & 6.2 & $25.42 \pm 1.12$ & $0.94 \pm 0.06$ & - & 0.35 & 2.23 & 2.57 & -0.34 \\
\hline & 617 & $\mathrm{CBP}$ & 86.4 & $23.74 \pm 1.14$ & $1.00 \pm 0.09$ & - & 1.89 & 2.01 & 2.36 & -0.35 \\
\hline \multirow{2}{*}{$10 \mathrm{~g}$} & 490 & Zeonex & 2.0 & $4.73 \pm 0.38$ & $0.96 \pm 0.08$ & - & 0.38 & 2.53 & 2.55 & -0.02 \\
\hline & 511 & CBP & 29.3 & $7.80 \pm 0.17$ & $0.58 \pm 0.02$ & $0.72 \pm 0.07$ & 0.18 & 2.43 & 2.22 & 0.20 \\
\hline \multirow{2}{*}{$10 \mathrm{~h}$} & 513 & Zeonex & 49.5 & $8.22 \pm 0.88$ & $3.99 \pm 0.41$ & - & 3.90 & 2.42 & 2.17 & 0.25 \\
\hline & 507 & CBP & 36.7 & $9.41 \pm 0.24$ & $1.60 \pm 0.11$ & - & 5.30 & 2.44 & 2.15 & 0.29 \\
\hline \multirow{2}{*}{$10 \mathrm{i}$} & 536 & Zeonex & 0.4 & $8.26 \pm 0.25$ & $1.03 \pm 0.12$ & $1.60 \pm 0.04$ & 14.40 & 2.31 & 2.34 & -0.03 \\
\hline & 517 & $\mathrm{CBP}$ & 9.2 & $7.42 \pm 0.18$ & $1.22 \pm 0.08$ & - & 10.02 & 2.40 & 2.27 & 0.12 \\
\hline \multirow{2}{*}{$10 \mathrm{j}$} & 525 & Zeonex & 18.1 & $10.02 \pm 0.42$ & $2.78 \pm 0.37$ & - & 3.05 & 2.36 & 2.07 & 0.29 \\
\hline & 500 & CBP & 19.1 & $5.35 \pm 0.14$ & $12.98 \pm 0.92$ & - & 0.32 & 2.48 & 2.24 & 0.24 \\
\hline \multirow{2}{*}{$10 \mathrm{k}$} & 511 & Zeonex & 16.7 & $7.74 \pm 0.75$ & $1.20 \pm 0.13$ & - & 3.06 & 2.42 & 2.13 & 0.29 \\
\hline & 508 & CBP & 18.9 & $5.83 \pm 0.21$ & $1.75 \pm 0.18$ & - & 0.94 & 2.44 & 2.19 & 0.25 \\
\hline \multirow{2}{*}{101} & 495 & Zeonex & 12.4 & $3.10 \pm 0.13$ & $0.66 \pm 0.05$ & $6.70 \pm 0.73$ & 4.36 & 2.50 & 2.18 & 0.32 \\
\hline & 500 & $\mathrm{CBP}$ & 16.2 & $7.57 \pm 0.09$ & $1.31 \pm 0.11$ & - & 2.07 & 2.48 & 2.21 & 0.27 \\
\hline \multirow{2}{*}{$10 \mathrm{~m}$} & 512 & Zeonex & 1.0 & $3.07 \pm 0.31$ & $1.58 \pm 0.14$ & $3.12 \pm 0.23$ & 0.73 & 2.42 & 2.11 & 0.31 \\
\hline & 503 & CBP & 17.4 & $5.57 \pm 0.36$ & $0.79 \pm 0.08$ & - & 65.43 & 2.46 & 2.09 & 0.37 \\
\hline \multirow{2}{*}{$10 n$} & 546 & Zeonex & 2.1 & $4.05 \pm 0.02$ & $1.98 \pm 0.18$ & - & 13.14 & 2.12 & 2.04 & 0.08 \\
\hline & 532 & $\mathrm{CBP}$ & 29.6 & $1.03 \pm 0.08$ & $0.86 \pm 0.06$ & - & 62.10 & 2.33 & 2.06 & 0.26 \\
\hline
\end{tabular}

a Photoluminescence maximum; ${ }^{b}$ Photoluminescence quantum yield; ${ }^{c}$ Prompt fluorescence lifetime in the host; ${ }^{d}$ Delayed emission lifetime in the host; ${ }^{e}$ Delayed fluorescence (DF) to prompt fluorescence (PF) ratio in the host; ${ }^{\mathrm{f}}$ Singlet and triplet energy in host. Error $\pm 0.03 \mathrm{eV} ;{ }^{\mathrm{g}}$ Singlet-triplet energy splitting in Zeonex. Error $\pm 0.05 \mathrm{eV}$.

The characteristics of the OLED devices revealed a significant increase in OLED efficiency depending on the structure (Figure 9b,e). The device based on emitter $10 \mathrm{~h}$ with trifluoromethyl group was found to be the most efficient with external quantum efficiency around 11.5\% (Figure 9b). Also, about 10\% EQE was obtained for the device based on emitter $10 \mathrm{f}$ with double nitrile groups and singlet-triplet inversion effect. The highest RTP based OLED was obtained for devices based on emitter $10 \mathrm{~g}$ with thiophene unit, up to $3.1 \%$. The highest luminance was obtained for the OLED based on emitter 10f, up to 24,680 $\mathrm{cd} / \mathrm{m}^{2}$, whereas RTP based OLED (10g), up to 10,250 $\mathrm{cd} / \mathrm{m}^{2}$ (Figure 9c). If we think about structure impact on the final device, the additional benzene ring allows increasing the EQE 2.5 times (10a, 10b). The insertion of the nitrogen group resulted in double increase of EQE $(10 \mathrm{a}, 10 \mathrm{e})$ and four fold with the additional bromine group (10a, $10 \mathrm{n})$. Pure halogen groups had only a limited impact on the increase of the overall efficiency (lower than $2 \mathrm{x}$ ).

\section{Conclusions}

We have successfully accomplished the synthesis of a unique, phenazine terminated aza-bowled polycyclic aromatic hydrocarbons which were identified as interesting group of novel emitters. The envisaged curvature was unambiguously confirmed by the X-ray crystallographic analysis. This revealed the desired intermolecular D $\cdots A$ interactions, which greatly influence their optoelectronic characteristic. Further examination of physicochemical properties, in conjunction with quantum calculations, pointed out the dependency between phenazine side group or the nitrogen atom insertion, at the significant change in the emission properties. The later ones were manifested by the excellent photoluminescence quantum yields (up to $86.4 \%$ ) and the controlled change between thermally activated delayed fluorescence (TADF) or room-temperature phosphorescence (RTP). The largest device throughput among all fabricated was recognized to the emitter containing dinitrile terminated species, resulting in a very high efficiency (>11\%) and a very efficient TADF process. 

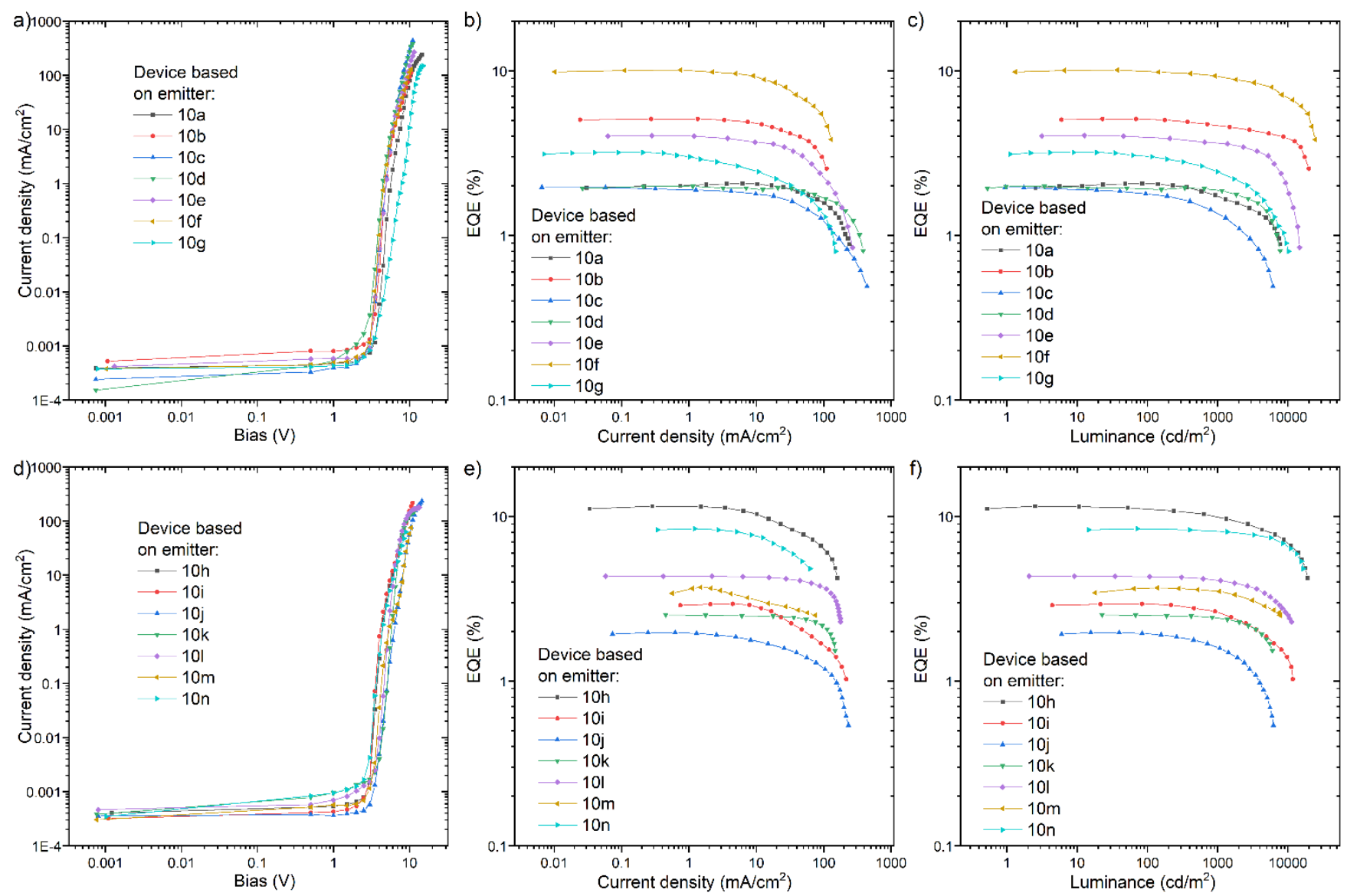

Figure 9. The characteristics of the OLED devices based on emitters 10a-n. a), d) Current density-bias characteristics. b), e) EQEcurrent density characteristics. c), f) EQE - luminance characteristics.

These studies provided a new, conceptually simple, and synthetically affordable, approach towards D-A curved NPAHs systems with excellent optoelectronic properties to be utilized as a future functional material. The established strategy opens an avenue for tuning the photo and redox properties of non-planar N-PAHs through rational vertical and/or horizontal synthetic modifications. Such approach could be also aimed to justify negative $\Delta \mathrm{E}_{\mathrm{ST}}$ and therefore it is in progress in our laboratory.

\section{ASSOCIATED CONTENT}

This material is available free of charge via the Internet at http://pubs.acs.org."

Accession Code CCDC 2128847 contains the supplementary crystallographic data for this paper. These data can be obtained free of charge via www.ccdc.cam.ac.uk/data_request/cif, or by emailing data_request@ccdc.cam.ac.uk, or by contacting The Cambridge Crystallographic Data Centre, 12 Union Road, Cambridge CB2 1EZ, UK; fax: +44 1223336033.

\section{AUTHOR INFORMATION}

\section{Corresponding Author}

* Adam Kubas - Institute of Physical Chemistry, Polish Academy of Sciences, Kasprzaka 44/52, 01-224
Warsaw, Poland; https://orcid.org/0000-0002-5508-0533; akubas@ichf.edu.pl

* Przemysław Data - Silesian University of Technology, Faculty of Chemistry, Department of Physical Chemistry and Technology of Polymers, Strzody 9/208d, 44-100 Gliwice, Poland; https://orcid.org/0000-0002-1831971X przemyslaw.data@polsl.pl

* Marcin Lindner - Institute of Organic Chemistry, Polish Academy of Sciences, Kasprzaka 44/52, 01-224 Warsaw, Poland; https://orcid.org/0000-0002-5514-674X; mlindner@icho.edu.pl

\section{Authors}

Jakub Wagner - Institute of Organic Chemistry, Polish Academy of Sciences, Kasprzaka 44/52, 01-224 Warsaw, Poland; https://orcid.org/0000-0002-5514-674X; mlindner@icho.edu.pl

Paola Zimmermann Crocomo - Silesian University of Technology, Faculty of Chemistry, Department of Physical Chemistry and Technology of Polymers, Strzody 9/208d, 44-100 Gliwice, Poland; https://orcid.org/00000002-1831-971X, paola.crocomo@polsl.pl

Michał Kochman - Institute of Physical Chemistry, Polish Academy of Sciences, Kasprzaka 44/52, 01-224 
Warsaw, Poland; https://orcid.org/0000-0003-2552-

9464; mkochman@ichf.edu.pl

\section{Author Contributions}

‡J.W., J.Z.C., M.K. contributed equally

\section{Funding Sources}

\section{National Science Centre, Poland, Grant No. UMO- 2018/31/D/ST5/00426}

National Science Centre, Poland, Grant No. 2020/39/B/ST4/01952

EU H2020 MSCA grant agreement No. 847413

Minister of Science and Higher Education of Poland "PMW" program agreement no. 5005/H2020-MSCA-COFUND/2019/2 Polish National Science Centre funding, grant no. 2017/25/B/ST5/02488.

EU H2020 ERA-Chair project ExCEED, grant agreement No 952008.

\section{Notes}

The authors declare no competing financial interest.

\section{ACKNOWLEDGMENT}

J.W. and M.L acknowledge support from the National Science Centre, Poland, Grant No. UMO-2018/31/D/ST5/00426

M.L. is a recipient of a scholarship awarded by the Polish Ministry of Education and Science to outstanding young scientists.

M. A. K. acknowledges funding from the European Union's Horizon 2020 research and innovation program under the Marie Skłodowska-Curie grant agreement No. 847413. A. K. acknowledges support from the National Science Centre, Poland, Grant No. 2020/39/B/ST4/01952.

This work has been published as part of an international cofinanced project funded from the program of the Minister of Science and Higher Education entitled "PMW" in the years 2020-2024; agreement no. 5005/H2020-MSCACOFUND/2019/2.

P.D. and P.Z.C acknowledges the Polish National Science Centre funding, grant no. 2017/25/B/ST5/02488. P.D. and P.Z.C acknowledges the supporting awards from the Rector of the Silesian University of Technology (04_040_SDU_10-2204, 04/040/RGJ21/0149). P.D. and P.Z.C. acknowledges the supporting actions from EU's Horizon 2020 ERA-Chair project ExCEED, grant agreement No 952008.

We are grateful to Mr Jan-Simon von Glasenapp of the Otto Diels-Institute for Organic Chemistry at the ChristianAlbrechts-University of Kiel for providing us with a copy of the ACID software package and helpful instructions on compiling and using that program.

\section{REFERENCES}

(1) Narita, A.; Wang, X.-Y.; Feng, X.; Müllen, K. New Advances in Nanographene Chemistry. Chemical Society Reviews 2015, 44 (18), 6616-6643.

(2) Stępień, M.; Gońka, E.; Żyła, M.; Sprutta, N. Heterocyclic Nanographenes and Other Polycyclic Heteroaromatic Compounds: Synthetic Routes, Properties, and Applications. Chemical Reviews 2017, 117 (4), 3479-3716.
Borissov, A.; Maurya, Y. K.; Moshniaha, L.; Wong, W.-S.; Żyła-Karwowska, M.; Stępień, M. Recent Advances in Heterocyclic Nanographenes and Other Polycyclic Heteroaromatic Compounds. Chemical Reviews 2021, acs.chemrev.1c00449;doi.org/10.1021/acs.chemrev.1c0 0449 .

Bachar, N.; Liberman, L.; Muallem, F.; Feng, X.; Müllen, K.; Haick, H. Sensor Arrays Based on Polycyclic Aromatic Hydrocarbons: Chemiresistors versus Quartz-Crystal Microbalance. ACS Applied Materials \& Interfaces 2013, 5 (22), 11641-11653.

Miao, Q. Ten Years of N-Heteropentacenes as Semiconductors for Organic Thin-Film Transistors. Advanced Materials 2014, 26 (31), 5541-5549.

Aumaitre, C.; Morin, J. Polycyclic Aromatic Hydrocarbons as Potential Building Blocks for Organic Solar Cells. The Chemical Record 2019, 19 (6), 1142-1154.

Hong, G.; Diao, S.; Antaris, A. L.; Dai, H. Carbon Nanomaterials for Biological Imaging and Nanomedicinal Therapy. Chemical Reviews 2015, 115 (19), 1081610906

(8) Weil, T.; Vosch, T.; Hofkens, J.; Peneva, K.; Müllen, K. The Rylene Colorant Family-Tailored Nanoemitters for Photonics Research and Applications. Angewandte Chemie International Edition 2010, 49 (48), 9068-9093.

(9) Jung, C.; Müller, B. K.; Lamb, D. C.; Nolde, F.; Müllen, K.; Bräuchle, C. A New Photostable Terrylene Diimide Dye for Applications in Single Molecule Studies and Membrane Labeling. Journal of the American Chemical Society 2006, 128 (15), 5283-5291.

(10) Schmidt-Mende, L.; Fechtenkötter, A.; Müllen, K.; Moons, E.; Friend, R. H.; MacKenzie, J. D. Self-Organized Discotic Liquid Crystals for High-Efficiency Organic Photovoltaics. Science 2001, 293 (5532), 1119-1122.

(11) Klaus Mullen; Ullrich Scherf. Organic Light Emitting Devices: Synthesis, Properties and Applications. WileyVCH: Weinheim. 2006.

(12) Hirai, M.; Tanaka, N.; Sakai, M.; Yamaguchi, S. Structurally Constrained Boron-, Nitrogen-, Silicon-, and PhosphorusCentered Polycyclic $\pi$-Conjugated Systems. Chemical Reviews 2019, 119 (14), 8291-8331.

(13) Schaub, T. A.; Padberg, K.; Kivala, M. Bridged Triarylboranes, -silanes, -amines, and -phosphines as Minimalistic Heteroatom-containing Polycyclic Aromatic Hydrocarbons: Progress and Challenges. Journal of Physical Organic Chemistry 2020, 33 (2).

(14) Kuratsu, M.; Kozaki, M.; Okada, K. 2,2':6',2":6",6Trioxytriphenylamine: Synthesis and Properties of the Radical Cation and Neutral Species. Angewandte Chemie International Edition 2005, 44 (26), 4056-4058

(15) Gilman, H.; Stuckwisch, C. G. The Di-Metalation of 9Phenylcarbazole. Journal of the American Chemical Society 1943, 65 (9), 1729-1733

(16) Kato, S. ichiro; Matsuoka, T.; Suzuki, S.; Asano, M. S.; Yoshihara, T.; Tobita, S.; Matsumoto, T.; Kitamura, C. Synthesis, Structures, and Properties of Neutral and Radical Cationic s,c,c-Bridged Triphenylamines. Organic Letters 2020, 22 (2), 734-738

(17) Zou, S. N.; Peng, C. C.; Yang, S. Y.; Qu, Y. K.; Yu, Y. J.; Chen, X.; Jiang, Z. Q.; Liao, L. S. Fully Bridged Triphenylamine Derivatives as Color-Tunable Thermally Activated Delayed Fluorescence Emitters. Organic Letters 2021, 23 (3), 958-962

(18) Ito, S.; Tokimaru, Y.; Nozaki, K. Benzene-Fused Azacorannulene Bearing an Internal Nitrogen Atom. An- 
gewandte Chemie International Edition 2015, 54 (25), 7256-7260

(19) Yokoi, H.; Hiraoka, Y.; Hiroto, S.; Sakamaki, D.; Seki, S.; Shinokubo, H. Nitrogen-Embedded Buckybowl and Its Assembly with C60. Nature Communications 2015, 6 (1), 8215-

(20) Deng, N.; Zhang, G. Nitrogen-Centered Concave Molecules with Double Fused Pentagons. Organic Letters 2019, 21 (13), 5248-5261

(21) Song, Y.; Zhang, G. Effect of Fusion Manner of Concave Molecules on the Properties of Resulting Nanoboats. Organic Letters 2021, 23 (2), 491-496

(22) Zhou, L.; Zhang, G. A Nanoboat with Fused Concave NHeterotriangulene. Angewandte Chemie - International Edition 2020, 59 (23), 8963-8968

(23) Zhu, G.; Song, Y.; Zhang, Q.; Ding, W.; Chen, X.; Wang, Y.; Zhang, G. Modulating the Properties of Buckybowls Containing Multiple Heteroatoms. Organic Chemistry Frontiers 2021, 8 (4), 727-735

(24) Mishra, S.; Krzeszewski, M.; Pignedoli, C. A.; Ruffieux, P.; Fasel, R.; Gryko, D. T. On-Surface Synthesis of a NitrogenEmbedded Buckybowl with Inverse Stone-ThrowerWales Topology. Nature Communications 2018, 9 (1), 1714

(25) Krzeszewski, M.; Dobrzycki, Ł.; Sobolewski, A. L.; Cyrański, M. K.; Gryko, D. T. Bowl-Shaped Pentagon- and Heptagon-Embedded Nanographene Containing a Central Pyrrolo[3,2-b]Pyrrole Core. Angewandte Chemie - International Edition 2021, 60 (27), 14998-15005

(26) Yang, Z.; Mao, Z.; Xie, Z.; Zhang, Y.; Liu, S.; Zhao, J.; Xu, J.; Chi, Z.; Aldred, M. P. Recent Advances in Organic Thermally Activated Delayed Fluorescence Materials. Chemical Society Reviews 2017, 46 (3), 915-1016

(27) Wong, M. Y.; Zysman-Colman, E. Purely Organic Thermally Activated Delayed Fluorescence Materials for Organic Light-Emitting Diodes. Advanced Materials 2017, 29 (22), 1605444

(28) Chen, X.-K.; Kim, D.; Brédas, J.-L. Thermally Activated Delayed Fluorescence (TADF) Path toward Efficient Electroluminescence in Purely Organic Materials: Molecular Level Insight. Accounts of Chemical Research 2018, 51 (9), 2215-2224.

(29) Liu, Y.; Li, C.; Ren, Z.; Yan, S.; Bryce, M. R. All-Organic Thermally Activated Delayed Fluorescence Materials for Organic Light-Emitting Diodes. Nature Reviews Materials 2018, 3 (4), 18020.
(30) Data, P.; Takeda, Y. Recent Advancements in and the Future of Organic Emitters: TADF- and RTP-Active Multifunctional Organic Materials. Chemistry - An Asian Journal 2019, 14 (10), 1613-1636.

(31) Kukhta, N. A.; Bryce, M. R. Dual Emission in Purely Organic Materials for Optoelectronic Applications. Materials Horizons 2021, 8 (1), 33-55.

(32) Kricka, L. J.; Ledwith, A. Dibenz[b,f]Azepines and Related Ring Systems. Chemical Reviews 1974, 74 (1), 101-123.

(33) Wu, Y.; Jin, Y.; Xu, J.; Lv, Y.; Yu, J. Recent Progress in the Synthesis and Applications of Azaacenes. Current Organic Chemistry 2020, 24 (8), 885-899

(34) Surry, D. S.; Buchwald, S. L. Dialkylbiaryl Phosphines in Pd-Catalyzed Amination: A User's Guide. Chem. Sci. 2011, 2 (1), 27-50

(35) Steinberg, B. D.; Jackson, E. A.; Filatov, A. S.; Wakamiya, A.; Petrukhina, M. A.; Scott, L. T. Aromatic $\pi$-Systems More Curved Than $\mathrm{C}_{60}$. The Complete Family of All Indenocorannulenes Synthesized by Iterative MicrowaveAssisted Intramolecular Arylations. Journal of the American Chemical Society 2009, 131 (30), 10537-10545.

(36) Hu, J.; Zhang, D.; Harris, F. W. Ruthenium(III) Chloride Catalyzed Oxidation of Pyrene and 2,7-Disubstitued Pyrenes: An Efficient, One-Step Synthesis of Pyrene-4,5Diones and Pyrene-4,5,9,10-Tetraones. The Journal of Organic Chemistry 2005, 70 (2), 707-708.

(37) G. Back, T. Oxidations Catalyzed By Seleninic Acids and Anhydrides, Their Precursors and Congeners. Current Green Chemistry 2016, 3 (1), 76-91.

(38) Trofimov, A. B.; Schirmer, J. An Efficient Polarization Propagator Approach to Valence Electron Excitation Spectra. Journal of Physics B: Atomic, Molecular and Optical Physics 1995, 28 (12), 2299-2324.

(39) Köhn, A.; Hättig, C. Analytic Gradients for Excited States in the Coupled-Cluster Model CC2 Employing the Resolution-of-the-Identity Approximation. Journal of Chemical Physics 2003, 119 (10).

(40) Martin, R. L. Natural Transition Orbitals. Journal of Chemical Physics 2003, 118 (11), 4775

(41) Dias, F. B.; Penfold, T. J.; Monkman, A. P. Photophysics of Thermally Activated Delayed Fluorescence Molecules. Methods and Applications in Fluorescence. 2017. 5, 012001 